\title{
Efecto de la oxitocina versus misoprostol en la disminución del sangrado y duración del alumbramiento, en gestantes atendidas en el Hospital Tingo María Minsa.
} Effect of oxytocin versus misoprostol in reducing bleeding and duration of third, in pregnant women
treated in Hospital Tingo María Minsa.

Hilda F. Falcón Condezo ${ }^{1}$

\section{RESUMEN}

Objetivo: Determinar el efecto de la oxitócina y misoprostol en la disminución del sangrado y duración del período de alumbramiento, en gestantes atendidas en el Hospital Tingo María, año 2008. Materiales y Métodos: Es un estudio cuasiexperimental, no aleatorizado, con dos grupos uno de estudio y otro de comparación; prospectivo, las pacientes fueron incluidas a partir del momento en que se decide el comienzo del estudio hacia delante; comparativo, porque se contrasto el nivel de eficacia de los fármacos oxitocina versus misoprostol, en el disminución del sangrado y duración del período de alumbramiento. Para el procesamiento y análisis estadístico se utilizó el programa SPSS versión 15.0 utilizándose la prueba Z y regresión logística para contrastar la hipótesis. Resultados: No existe diferencia estadísticamente significativa ( $\mathrm{p}>0.05)$ entre los promedios de volumen de sangrado después de la intervención entre los casos y controles. Existe diferencia estadísticamente significativa $(\mathrm{p}<0.05)$ entre la duración del periodo de alumbramiento después de la intervención entre los casos y controles. Conclusión: El misoprostol administrado vía sublingual $(100 \mathrm{mcg})$ no ha demostrado ser superior a la oxitocina administrada vía intramuscular (10 UI), en la disminución del volumen del sangrado vaginal; el misoprostol administrado vía sublingual $(100 \mathrm{mcg})$ ha demostrado ser superior a la oxitocina administrada vía intramuscular (10 UI), en la disminución del tiempo de alumbramiento en mujeres en trabajo de parto atendidas en el Hospital Tingo María, por tanto es un alternativa eficaz para el manejo activo del periodo de alumbramiento.

Palabra clave: Misoprostol - Oxitocina - Sangrado vaginal - Tiempo de alumbramiento (Fuente: BIREME)

\begin{abstract}
Objective: To determine the efecct oxytocin and misoprostol in the reducing bleeding and duration of delivery in pregnant Assisted of Tingo Maria Hospital, year 2008. Materials and Methods: A quasi-experimental study, not randomized to one of two groups and a comparison study, prospective patients were included from the moment that decided the inception of forward; comparison, because it contrasts the level of effectiveness of misoprostol versus oxytocin drugs in the reduction of bleeding and length of confinement. For processing and statistical analysis was used SPSS version 15.0 using the $\mathrm{Z}$ test and logistic regression to test the hypothesis. Results: No statistically significant difference ( $>0.05$ ) between the average volume of bleeding after surgery among cases and controls. There is a statistically significant difference $(p<0.05)$ between the duration of confinement after the intervention between cases and controls. Conclusion: Misoprostol administered sublingually (100 mcg) has not proven superior to oxytocin intramuscularly (10 IU), in reducing the volume of bleeding vaginal; misoprostol given sublingually $(100 \mathrm{mcg})$ has proven superior to intramuscularly administered oxytocin (10 IU), the reduction in hours of delivery in laboring women treated at the Hospital Tingo Maria, so it is an effective alternative to active management of the delivery period.
\end{abstract}

Key words: Misoprostol - Oxytocin - Vaginal bleeding - Time of birth (source: BIREME)

\footnotetext{
${ }^{1}$ Mg. Salud Publica. Obstetriz Hospital Tingo María Minsa - Huánuco, Perú.
} 


\section{INTRODUCCIÓN}

La hemorragia post parto causa principal de mortalidad materna "representando a nivel Mundial el 24.8\%, en America Latina y el Caribe el 25\% en el Perú el 47\%; asimismo a nivel de la "Región Huánuco en los años 2000 a 2002 representa el 67\%, y a nivel de Tingo María el $60 \%$ de las muertes maternas fue por hemorragia post parto” (1). Más de la mitad de muertes suceden dentro de las 24 primeras horas después del parto, se deben a complicaciones que se presentan durante el alumbramiento; la mayoría de estos casos a consecuencia de una hemorragia masiva. Siendo las mujeres con anemia las más vulnerables. El parto es un factor que contribuye en la persistencia y severidad de esta patología. Esto último es de vital importancia, dada la prevalencia de casos de anemia severa entre las mujeres de los Países en desarrollo. La prevalencia de anemia en gestante peruanas es del 50\% (2), y la tasa de anemia en el Instituto Materno Perinatal es del 47.1\%, mientras en el Hospital de Tingo María el $10 \%$ de gestantes que acuden para la atención del parto son anémicas (3).

En el tercer período del parto, se produce la separación y la expulsión de la placenta. La mayor incidencia de hemorragia post parto ocurre durante el período de alumbramiento. En este periodo los músculos del útero se contraen y la placenta comienza a separarse de la pared uterina. Los volúmenes de sangre que se pierden dependen de la rapidez con que esto ocurra. El período del alumbramiento generalmente dura de 5 a 15 minutos y se considera que el tercer período del parto es prolongado, cuando supera este tiempo lo que indica una posible complicación (4).

En la atonía uterina los vasos sanguíneos en el sitio de la implantación de la placenta no se constriñen de manera adecuada, Lo que provoca un sangrado abundante.

Para la madre el principal riesgo es la hemorragia durante o después de la separación de la placenta, y la retención de la misma. La incidencia de la hemorragia post parto y de retención de la placenta se incrementa si existen factores que predisponen a ello, como el polihidramnios, un embarazo múltiple, un parto prolongado, la multiparidad, la macrosomía fetal etc. Y también ocurren con frecuencia si estas complicaciones están presentes en la historia obstétrica de la mujer. Por esto y hasta cierto punto, se puede determinar durante el embarazo y el parto qué mujeres tendrán un riesgo más elevado de desarrollar complicaciones en el alumbramiento. Pero incluso en embarazos de bajo riesgo con primera y segunda fase normal, pueden acontecer las citadas complicaciones. La forma en que se maneje el alumbramiento puede influenciar en la incidencia de complicaciones y en la cantidad de pérdida sanguínea.

La hemorragia post parto representa la pérdida excesiva de sangre proveniente del sitio de implantación placentaria, cuando ella se presenta, la hipovolemia concomitante puede poner en peligro la vida de la madre llevándole al shock y a la muerte en horas o exponerla a graves secuelas. Siendo la multiparidad un factor que condiciona a la hemorragia post parto por atonía uterina, es decir, a la falta de contracción apropiada del útero después del nacimiento del bebé. Como consecuencia de ello, no se controla el sangrado de los vasos sanguíneos en el útero y, si no se le presta la adecuada atención Obstétrica de inmediato, una mujer con hemorragia post parto tiene probabilidad de morir. La mayor cantidad de sangre que se pierde en el parto ocurre en el período de alumbramiento teniéndose como promedio de 500 $\mathrm{ml}$. este volumen es mayor en las multíparas que en las primíparas y en una operación cesárea aproximadamente de $1000 \mathrm{ml}$, en ambas circunstancias la pérdida hematica se produce fundamentalmente durante el alumbramiento y en la primera hora post parto. Muchas mujeres toleran bien está pérdida de sangre y no sufren de efectos adversos; sin embargo, los efectos varían según cada mujer, en el caso de las mujeres anémicas, la pérdida de incluso 200 a $250 \mathrm{ml}$ de sangre podría resultar fatal $(5,6)$.

La aplicación de este tratamiento en la zona de Tingo María sería beneficiosa en las madres gestantes multíparas, gran multíparas y anémicas siendo ellas la población de mayor riesgo de sufrir Hemorragia post parto. En consecuencia los intentos de disminuir el sangrado se deben realizar en este período, aplicando su manejo activo el cual consiste en una serie de intervenciones que se aplican para disminuir la pérdida sanguínea, acelerar la rápida expulsión de la placenta aumentando las contracciones uterinas, de esta manera previniendo la hemorragia post parto y evitando la atonía uterina.

Durante la atención del parto, se ha observado que la pérdida sanguínea de una a otra paciente es variable, en algunos casos el sangrado es profuso masivo y en otras es moderado, la cuantificación 
INVESTIGACIÓN ORIGINAL ORIGINAL RESEARCH
Efecto de la oxitocina versus misoprostol en la disminución del sangrado y duración del alumbramiento, en gestantes atendidas en el Hospital Tingo María Minsa.

Falcón H.

del sangrado no se hace con exactitud solo se calcula, casi siempre reportamos un volumen inferior al que tiene. Muchas veces nos damos con la sorpresa que estamos frente a un cuadro de shock hipovolémico percatándonos a través de la clínica (hipotensión, taquicardia, sudoración, palidez etc.) Asimismo se pudo observar cuadros críticos de hemorragia post parto por atonía uterina en pacientes gran multíparas que han terminado en histerectomías. La aplicación profiláctica de una droga uterotónica como la Oxitócica y Misoprostol para contraer el útero durante el período de alumbramiento previene tal pérdida sanguínea (6). El uso de esta tecnología sencilla salva las vidas de las mujeres en riesgo de hemorragia post parto.

Es probable que las mujeres que sobreviven a una hemorragia post parto sufran de anemia y de otras complicaciones. A menudo, ellas tienen que recibir transfusiones de sangre y son susceptibles de correr los riesgos relacionados con las reacciones o con la infección por VIH o la hepatitis. Con frecuencia, el sangrado que no se puede controlar utilizando medicamentos requiere cirugía, e incluso la histerectomía. Estos procedimientos son costosos y pueden resultar emocionalmente desoladores para la mujer y su familia. Además llevan aparejados el riesgo de infecciones, reacciones a la anestesia y otras complicaciones.

\section{MATERIALES Y METODOS}

Es un estudio Cuasiexperimental, no aleatorizado, con dos grupos, uno de estudio y otro de comparación. Prospectivo, las pacientes fueron incluidas a partir del momento en que se decide el comienzo del estudio hacia adelante. Comparativo, se comparó el efecto de los fármacos: Oxitocina y Misoprostol, en el disminución del sangrado y duración del período de alumbramiento.

\section{Calculo del tamaño muestral}

Se utilizó el muestreo a conveniencia, por tanto no fue necesario calcular el tamaño muestral, se incluyeron en el estudio 30 unidades de estudio para cada grupo a través de criterios de inclusión y exclusión.

Diseño gráfico del estudio

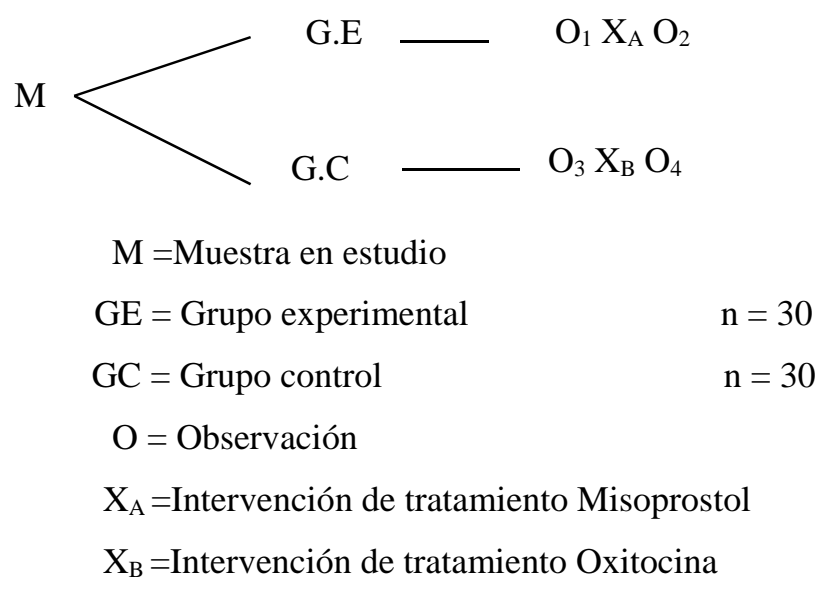

Criterios de Inclusión

El estudio se realizó en pacientes multigestas de alto riesgo de hemorragia post parto, que acuden para la atención del parto, con un inicio trabajo de parto espontáneo.

Criterios de exclusión
El estudio no se aplicó en aquellas gestantes que presenten factores de riesgo como:

- Cesárea previa

- Ruptura prematura de membranas prolongada

- Parto pre término

- Antecedentes de abortos recurrentes o habituales

- Óbito fetal.

- Embarazo prolongado 
INVESTIGACIÓN ORIGINAL ORIGINAL RESEARCH
Efecto de la oxitocina versus misoprostol en la disminución del sangrado y duración del alumbramiento, en gestantes atendidas en el Hospital Tingo María Minsa.
- Primigestas

- Gestantes con trabajo de parto incluido

- Amas, coagulopatía
- Desgarros severos del canal del parto

- Vertimiento accidental de agua, orina, heces etc.en el recipiente recolector de sangre.

Secuencia de procedimientos

\begin{tabular}{|c|c|}
\hline Grupo Estudio $\mathrm{n}=30$ & ión $\mathrm{n}=30$ \\
\hline $\begin{array}{l}\text {-Gestantes multigestas en trabajo de parto espontáneo } \\
\text { que acuden al Hospital Tingo María MINSA, } \\
\text { asignadas al grupo según criterios de inclusión } \\
\text { exclusión. } \\
\text { - Obtención del consentimiento informado } \\
\text { - Gestantes reciben Misoprostol } 100 \text { mcgr. Vía } \\
\text { sublingual. } \\
\text { - La administración del fármaco se hará durante el } \\
\text { período de alumbramiento en el momento de la salida } \\
\text { del hombro fetal. } \\
\text { - Extracción de la placenta mediante tracción firme y } \\
\text { controlada. } \\
\text { - Medición del sangrado con una jarra calibrada y con } \\
\text { paño control, desde el pinzamiento del cordón } \\
\text { umbilical hasta una hora después del parto (cuarto } \\
\text { periodo del parto). } \\
\text { - Medición del tiempo de duración del alumbramiento, } \\
\text { desde la administración del fármaco hasta la expulsión } \\
\text { de la placenta. } \\
\text { - Comparación de resultados. }\end{array}$ & $\begin{array}{l}\text { - Gestantes multigestas en trabajo de parto espontáneo } \\
\text { que acuden al Hospital Tingo María MINSA, } \\
\text { asignadas al grupo según criterios de inclusión } \\
\text { exclusión. } \\
\text { - Obtención del consentimiento informado } \\
\text { - Gestantes reciben Oxitocina } 10 \text { UI. Vía intramuscular. } \\
\text { - La administración del fármaco se hará durante el } \\
\text { período de alumbramiento en el momento de la salida } \\
\text { del hombro fetal. } \\
\text { - Extracción de la placenta mediante tracción firme y } \\
\text { controlada. } \\
\text { - Medición del sangrado con una jarra calibrada y con } \\
\text { paño control, desde el pinzamiento del cordón } \\
\text { umbilical hasta una hora después del parto (cuarto } \\
\text { periodo del parto). } \\
\text {-Medición del tiempo de duración del alumbramiento, } \\
\text { desde la administración del fármaco hasta la expulsión } \\
\text { de la placenta. } \\
\text { - Comparación de resultados. }\end{array}$ \\
\hline
\end{tabular}

Técnicas e instrumentos de recolección de datos:

La recolección de datos se hizo a través de la ficha de observación, en donde se consignaron los datos completos concernientes al estudio.

Procesamiento y análisis de los datos: Para procesar los datos se utilizó el software estadístico SPSS versión 15.0 para Windows y el análisis estadístico se hará con el Anova de dos vías y Newman-Keuls, esto es para establecer las diferencias en cuanto al sangrado y el tiempo de alumbramiento. Para las variables cualitativas se

\section{RESULTADOS}

El número total de participantes del estudio fue de 60 mujeres, de los cuales ingresaron al estudio un total de 30 pacientes al grupo misoprostol y 30 utilizará el método Chi cuadrado, se aceptaran resultados como significativos valores $\mathrm{p}=<0,05$.

Aspectos éticos.

El estudio fue conducido de acuerdo a la Declaración de Helsinki II y sus respectivas modificatorias incluido Edimburgo del año 2000. Los pacientes fueron informados en forma adecuada y de acuerdo al nivel sociocultural de cada uno de ellos del propósito del estudio y a todos se les solicito un consentimiento informado.

al grupo oxitocina, las características de la población incluida en el estudio, fueron comparables. 
INVESTIGACIÓN ORIGINAL ORIGINAL RESEARCH
Efecto de la oxitocina versus misoprostol en la disminución del sangrado y duración del alumbramiento, en gestantes atendidas en el Hospital Tingo María Minsa.

Tabla 1: Principales características de los grupos de estudio

\begin{tabular}{lll}
\hline Características & Misoprostol & Oxitocina \\
& $(\mathbf{n = 3 0 )}$ & $(\mathbf{n = 3 0 )}$ \\
\hline Edad (años) & 23.16 & 26.97 \\
Multíparas & $30(100 \%)$ & $30(100 \%)$ \\
Edad Gestacional (Sem) & 41.8 & 40.4 \\
\hline
\end{tabular}

Fuente: Proyecto MSPvsOXIT

Dentro del grupo de misoprostol se encontró que la media de las edades fue de 23.16 y en el grupo de oxitocina la media de las edades fue de 26.97; tanto en el grupo de misoprostol como el grupo de oxitocina el $100 \%$ de mujeres que ingresaron al estudio fueron multíparas y en el grupo de misoprostol se encontró que la media de la edad gestacional fue de 41.8 semanas y en el grupo de oxitocina la media de la edad gestacional fue de 40.4 semanas.

Hipótesis Alterna: El misoprostol tiene un mayor efecto en comparación con la oxitocina, en la disminución del sangrado y duración del período de alumbramiento en gestantes atendidas en el Hospital Tingo María, año 2008.

Hipótesis Nula: El misoprostol tiene un menor efecto en comparación con la oxitocina, en la disminución del sangrado y duración del período de alumbramiento en gestantes atendidas en el Hospital Tingo María, año 2008.

\section{Modelo lógico de contrastación}

\section{Si}

El misoprostol tiene un mejor efecto que la oxitocina en la disminución del sangrado y duración del período de alumbramiento en gestantes atendidas en el Hospital Tingo María, año 2008.

\section{Entonces}

La disminución del sangrado y la duración del periodo de alumbramiento de quienes reciben el misoprostol será menor que la observada en quienes reciben la oxitocina.

\section{Inferencia estadística}

Al evaluar la distribución de los datos, se concluye que no siguen una distribución normal, motivo por el cual se emplea la prueba no paramétrica de Mann-Whitney-Wilcoxon para identificar significancia estadística.

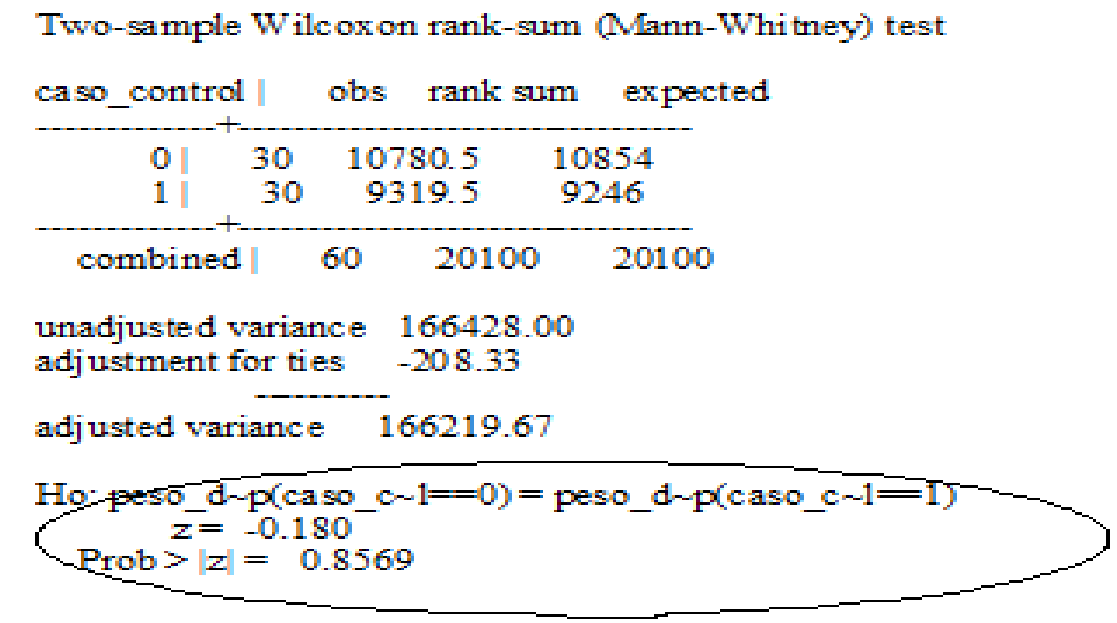

\section{Figura 1: Inferencia entre caso/control y disminución del sangrado después de la intervención} (Volumen del sangrado)

Conclusión: No existe diferencia estadísticamente significativa ( $p>0.05)$ entre los promedios de volumen de sangrado después de la intervención entre los casos y controles. 
INVESTIGACIÓN ORIGINAL ORIGINAL RESEARCH
Efecto de la oxitocina versus misoprostol en la disminución del sangrado y duración del alumbramiento, en gestantes atendidas en el Hospital Tingo María Minsa.

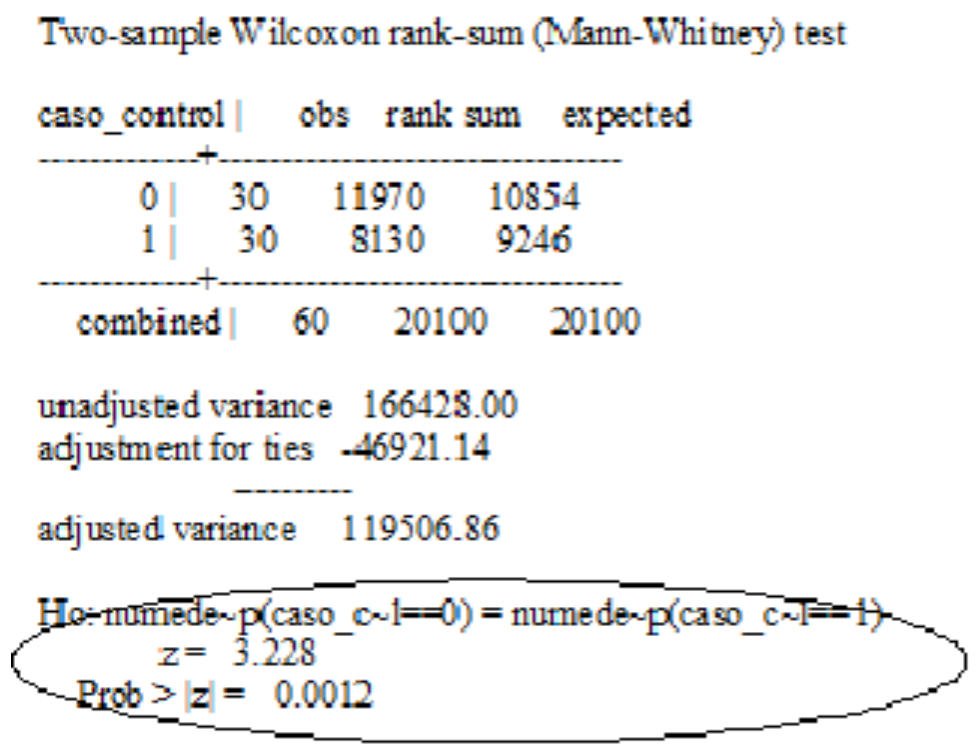

Figura 2: Inferencia entre caso/control duración del periodo de alumbramiento después de la intervención:

Conclusión: Existe diferencia estadísticamente significativa $(\mathrm{p}<0.05)$ entre la duración del periodo de alumbramiento después de la intervención entre los casos y controles.

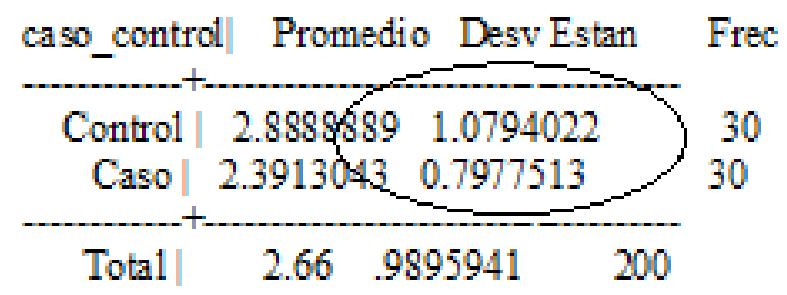

Figura 3: Promedio de duración del periodo de alumbramiento después de la intervención

Como se observa en la figura, los casos (con la intervención misoprostol) presentaron un menor tiempo de alumbramiento comparado con los que recibieron oxitocina, el cual es estadísticamente significativa $(\mathrm{p}<0.05)$.

Tabla 2: Variables con estadística significativa

\begin{tabular}{|c|c|c|c|c|c|c|}
\hline & \multicolumn{2}{|c|}{ Caso } & \multicolumn{2}{|c|}{ Control } & \multirow[t]{2}{*}{$\mathbf{z}$} & \multirow[t]{2}{*}{$\mathbf{p}$} \\
\hline & media & $\mathbf{S}$ & Media & $\mathbf{S}$ & & \\
\hline $\begin{array}{l}\text { Tiempo de alumbramiento después } \\
\text { de la Intervención }\end{array}$ & 2,39 & 0,79 & 2,89 & 1,08 & 3,228 & 0,0012 \\
\hline
\end{tabular}

Fuente: Proyecto MSPvsOXIT

El valor de la prueba $\mathrm{Z}$ demuestra disminución estadísticamente significativa del periodo de alumbramiento en el grupo de casos (intervención misoprostol), comparado con el grupo control (intervención oxitocina), siendo el valor $\mathrm{Z}$ de 3,228 y su valor $\mathrm{p}=0,0012$.
Por tanto se rechaza la hipótesis nula y se acepta la alterna en su item 02 que corresponde a la duración del periodo de alumbramiento de quienes reciben el misoprostol es menor que la observada en quienes reciben la oxitocina, diferencia 
INVESTIGACIÓN ORIGINAL ORIGINAL RESEARCH
Efecto de la oxitocina versus misoprostol en la disminución del sangrado y duración del alumbramiento, en gestantes atendidas en el Hospital Tingo María Minsa. estadísticamente significativa ( $<<0.05)$, mientras que respecto al item 01 que corresponde a la disminución del sangrado de quienes reciben el misoprostol es menor que la observada en quienes reciben la oxitocina, no hay diferencia estadísticamente significativa $(\mathrm{p}>0.05)$ entre los promedios de volumen de sangrado después de la intervención, entre los que reciben misoprostol y oxitocina.

En las ciencias sociales y básicamente en las ciencias de la salud se parte de la premisa de que el experimento no resultará en lo que desea demostrar. Se parte de premisas tal como: el tratamiento no es efectivo, no hay diferencia entre dos o más tratamientos.

\section{DISCUSIÓN}

El tratamiento convencional de primera línea de la hemorragia post parto de carácter no quirúrgico, incluyen el uso de medicamentos uterotónicos (como la oxitocina), la aplicación de masajes uterinos y la compresión bimanual. También pueden administrarse fluidos intravenosos 0 terapia con medicamentos y transfusiones sanguíneas para estabilizar a la paciente. Si con estas medidas iniciales no se logra controlar el sangrado, puede resultar necesario recurrir a intervenciones quirúrgicas de carácter avanzado.

En muchos países en desarrollo, los centros de atención primaria de salud y los centros de referencia no suelen contar con el personal médico, los insumos o el equipamiento necesarios para la administración rutinaria de los medicamentos uterotónicos convencionales. Generalmente los centros de salud tienen menos personal del necesario y es posible que el personal médico no esté calificado o disponible para administrar los medicamentos, aun cuando se disponga de éstos. Así también la oxitocina requiere refrigeración, condiciones especiales de almacenamiento y la utilización de agujas y jeringas elementos y condiciones que pueden no estar disponibles habitualmente en áreas de bajos recursos.

El misoprostol es un medicamento ampliamente disponible y regularmente indicado para prevenir las úlceras gástricas ocasionadas por el uso prolongado de medicamentos antinflamatorios no esteroides. Dada su capacidad de estimular las contracciones uterinas, el misoprostol ha sido utilizado durante más de una década para varias indicaciones de salud reproductiva (como la maduración cervical o la inducción del parto). El misoprostol también ha demostrado su potencial para prevenir y tratar HPP ocasionadas por atonía uterina. Distintos estudios
Según los datos observados en un experimento, que necesariamente contienen variabilidad, se toma la decisión de rechazar o no la premisa de la cual se partió. Si los datos observados llevan a la persona que investiga a rechazar la premisa de que, por ejemplo, el tratamiento no es efectivo, entonces ha demostrado que en efecto, el tratamiento es efectivo, en esta lógica es que se ha demostrado la hipótesis planteada en su segunda parte que corresponde a menor tiempo de alumbramiento, más no en la primera parte que corresponde a disminución del volumen de sangrado.

han evaluado el misoprostol como terapia alternativa, puesto que ofrece una serie de ventajas en comparación con el tratamiento convencional.

El misoprostol: puede ser administrado por diferentes vías (oral, rectal, sublingual, vaginal); no requiere refrigeración; permanece en buenas condiciones por tiempo prolongado; permanece estable en altas temperaturas; y sus efectos secundarios son relativamente escasos. Dadas estas características, el misoprostol resulta especialmente adecuado para ser empleado en países en desarrollo para el manejo de la hemorragia postparto. Puede ser utilizado por diferentes prestadores de salud en zonas de bajos recursos y administrarse eficazmente a nivel comunitario.

Miller en su revisión del manejo de la hemorragia uterina postparto en sitios de pocos recursos económicos concuerdan que el uterotónico más utilizado es la oxitocina sólo o en combinación con ergotamina, pero en los sitios donde no hubiere estos recursos, el misoprostol podría ser útil para el tratamiento de la hemorragia uterina aunque es menos efectivo que los medicamentos anteriores mencionados, resultado que coincide con lo encontrado en nuestro estudio respecto a la disminución del sangrado vaginal (11). En otro estudio clínico, randomizado, doble ciego compararon el uso de misoprostol oral versus el uso de sintometrina en el tratamiento de la hemorragia postparto. Encontraron que no hubo diferencia significativa en pérdida de sangre entre los dos grupos. En el grupo que recibió misoprostol hubo más mujeres que tuvieron necesidad de utilizar oxitócico (12).

Uddin en su estudio sobre el perfil farmacocinético y efectos adversos del misoprostol administrado por vía rectal en el tratamiento de la hemorragia postparto concluyen que el misoprostol administrado por vía rectal se asocia con niveles 
INVESTIGACIÓN ORIGINAL ORIGINAL RESEARCH
Efecto de la oxitocina versus misoprostol en la disminución del sangrado y duración del alumbramiento, en gestantes atendidas en el Hospital Tingo María Minsa. pico menores que los alcanzados por vía oral y que puede tener mayor eficacia al aumentar la dosis, pero disminuye su aceptabilidad al tratamiento (13). Por otro lado Caliskan et al (14), compararon el uso de misoprostol oral versus oxitocina versus misoprostol oral+oxitocina versus oxitocina+metilergonavina. Encontraron que la hemorragia postparto en el grupo de misoprostol y oxitocina fue similar, pero fue significativamente mayor en comparación a los otros grupos. Concluyen que el misoprostol oral tiene igual efectividad que la oxitocina, pero tiene menos eficacia en comparación con los grupos de oxitocina + misoprostol oral y oxitocina + metilergonavina; hallazgo que coincide con los resultados del presente estudio donde respecto a la disminución del sangrado no hay diferencia estadísticamente significativa $(p>0.05)$ entre los promedios de volumen de sangrado después de la intervención, entre los que recibieron misoprostol y oxitocina.

Otro estudio prospectivo, observacional y comparativo comparó el misoprostol vaginal con oxitocina. Reportando menor sangrado en el grupo de pacientes que utilizaron misoprostol y menor uso de transfusiones, pero esta diferencia no fue estadísticamente significativa. Concluyen que el misoprostol vaginal es efectivo para el control de la hemorragia postparto, pero cabe resaltar que su nivel de evidencia es menor al no haber aleatorización de los pacientes y no ser un estudio ciego (15).

Por otro lado en el año 2000 la razón de mortalidad materna a nivel nacional en el Perú, se halló en 164.2 por cien mil nacidos vivos; la hemorragia puerperal representa el 54.7\%. La mortalidad materna en el puerperio fue de $40.3 \%$ según el estudio "Tendencia, niveles y estructura de la mortalidad materna en el Perú 1992 - 2000” y para el ENDES del año 2000 la prevalencia ascendía a 185.0 por cien mil nacidos vivos; debido a este problema de salud pública es que es ineludible el tomar medidas que impidan y/o controlen la hemorragia post parto, hallándose dentro de ella el manejo activo del periodo de alumbramiento como una de las estrategias más importantes, siendo los fármacos más utilizados el misoprostol y la oxitocina, en este contexto los resultados del presente estudio coinciden con los hallazgos del estudio conducido por Angarita (16), quienes demostraron que la administración de 100Ug de misoprostol sublingual en el tercer periodo del parto reduce el tiempo de alumbramiento, lo cual es concordante con el hallazgo nuestro respecto a la duración del periodo de alumbramiento de quienes reciben el misoprostol es menor que la observada en quienes reciben la oxitocina, diferencia estadísticamente significativa $(\mathrm{p}<0.05)(17,18,19,20$.

Concluyendo:

- El misoprostol administrado vía sublingual (100 mcg) no ha demostrado ser superior a la oxitocina administrada vía intramuscular (10 UI), en la disminución del volumen del sangrado vaginal en mujeres en trabajo de parto atendidas en el Hospital Tingo María.

- El misoprostol administrado vía sublingual (100 mcg) ha demostrado ser superior a la oxitocina administrada vía intramuscular (10 UI), en la disminución del tiempo de alumbramiento en mujeres en trabajo de parto atendidas en el Hospital Tingo María, por tanto es un alternativa eficaz para el manejo activo del periodo de alumbramiento.

Debemos de manifestar algunas limitaciones del estudio al interpretar los hallazgos: no evaluamos algunos variables tales como frecuencia de reacciones adversas o eventos estresantes de la vida, que pudieran influir en el proceso de atención del parto y el puerperio y su culminación, siendo importante llevar a cabo otros estudios con un mayor número de mujeres y con un diseño doble ciego.

\section{AUTOR DE CORRESPONDENCIA}

Hilda F. Falcón Condezo

Hospital Tingo María Minsa. Rupa Rupa, Leoncio Prado, Huánuco, Perú.

E-mail: hildauras5@yahoo.es

\section{REFERENCIAS BIBLIOGRAFICAS}

1. Boletín Pathfinder Internacional. “Organización Mundial de la Salud Mortalidad Materna por Hemorragia Post parto”. 2000.

2. Instituto Nacional de Nutrición. "Anemia en gestantes peruanas”.1992.

3. PSMPN-HTM .Registros de informes estadísticos .Anemia .2003

4. Prendiville, W.J. et al. Active versus expectant management in the trird stage of labour (Cochrane Review, March 9, 2000). In: The Cochrane Library, Issue 4, 2001. Oxford: Update Software, Ltd.

5. Hospital San Bartolomé .Manual Atención Integral de la Salud Perinatal, Pág. 58-64. 2003, Lima -Perú.

6. Cunninghan, F.G.et al. Williams Obstetrics.20 th ed. Stamford, Connecticut: Appleton versus Lange (1997).

7. Gulmezoglu; a.m. Personal Communication, WHO/August 28, 2001). 
8. Oréstedes Cabanillas Sánchez. 2007. Tesis de Especialista en Ginecoobstetricia UNMSM, Eficacia del misoprostol sublingual en el alumbramiento dirigido.

9. Canettí Marl, Silvia; Nuñez Alarcon, Maribel. Alumbramiento dirigido con oxitocina o metilergobasina. Caracas; s.n; sept. 1997.18 p. ilus, tab.

10. Angarita W, Borre O, Rodríguez B. Manejo activo del alumbramiento con misoprostol sublingual: un estudio clínico controlado en el Hospital de Maternidad Rafael Calvo de Cartagena. Rev. colomb. obstet. ginecol;53(1):87-91, ene.-mar. 2002. tab.

11. Miller S, Lester F, Hensleigh $P$. Prevention and treatment of postpartum hemorrhage: new advances for lowresource settings. J Midwifery Womens Health. 2004 Jul-Aug;49(4):283-92.

12. G. Justus Hofmeyr, Gijs Walraven, A. Metin Gülmezoglu, Babalwa Maholwana, Zarko Alfirevic, Jose Villar. (2005) Misoprostol to treat postpartum haemorrhage: a systematic review. BJOG: An International Journal of Obstetrics and Gynaecology 112:5, 547-553.

13. Uddin, Khan Ru, El-Refaey $H$. Pharmacokinetics and adverse-effect profile of rectally administered misoprostol in the third stage of labor. Obstet Gynecol. 2003 May;101(5 Pt 1):968-74.

14. Caliskan E, Dilbaz B, Meydanli MM, Ozturk N, Narin MA, Haberal A. Oral misoprostol for the third stage of labor: a randomized controlled trial. Obstet Gynecol. 2003 May;101(5 Pt 1):921-8.

15. Quiroga R, Esparza M, Batiza V, Coronado O, Hernández S, Martínez J. Misoprostol Vaginal para la Prevención de la Hemorragia Postparto. Ginecología y Obstetricia de México. 2002; vol 70(11):572-575.

16. Angarita W, Borre O, Rodríguez B. Misoprostol sublingual en el tercer periodo del parto estudio clínico doble ciego, con asignación aleatoria, controlado con placebo. Rev Col Obst Gin 2003; 54 (2): 81-86.

17. Begley, C.A Comparison of 'active and physiological 'management of the third stage of labour. Midwifery 6:3 - 17 (1990).

18. Bulgaho, A Et al. Misoprostol for prevention of post partum hemorrhage. International Journal of Gynecology versus Obstetrics 73:1 - 6 (2001).
19. Poeschmann, R.P. Et al. A randomized Comparison of oxytocin, Sulprostone and placebo in the management of the third stage of Labour. Bristish journal of Obstetrics versus Gynecology 98. 528 530 (1991).

20. Nordstrom, L. E t al. Routine oxytocin in the third stage of labour. A Placebo controlled randomised trial. British Journal of Obstetrics versus Gynecology 104: 781 - 786 (1997).

21. USAID. Oficina de Salud y Nutrición Centro para la población, la Salud y la Nutrición, Dirección para programas Mundiales.

22. Libro de registro de Partos. Servicio de Obstetricia. Hospital Tingo Maria, Folio $\mathrm{N}^{\mathrm{a}}$ 0482-0535 (2003).

23. Khan, G.Q.et al. Controlled Cord traction versus minimal intervención Techniques in delivery of the placenta: a randomized Controlled trial. American Journal of Obstetrics and Gynecology 177: 700 - 774 (1997).

24. Rogers, J. et al. Active versus expectant management of third stage of Third stage of labor: the Hinchingbrooke randomized controlled trial Lancet 351: 693 - 699 (1998).Base Cochrane y Biblioteca de Salud Reproductiva de la OMS.

25. Shuartz, Ricardo "Tratado de Obstetricia” Ed. El ateneo, 5ta Edición. 1,998, Argentina.

26. Salud Perinatal - CLAP - N 176.

27. Revista Aniversario. Unidad Territorial de Salud Tingo María. Pag. 6 - 29 .Mayo 1995.

23. Starrs, A. The Safe Motherhood Agenda: Prioritis for the Next Decade. New York: Inter-Agency Group for Safe Motherhood, Family Care International (1997).

28. G. Justus Hofmeyr, Gijs Walraven, A. Metin Gülmezoglu, Babalwa Maholwana, Zarko Alfirevic, Jose Villar. (2005) Misoprostol to treat postpartum haemorrhage: a systematic review. BJOG: An International Journal of Obstetrics and Gynaecology 112:5, 547-553.

29. Uddin, Khan Ru, El-Refaey $H$. Pharmacokinetics and adverse-effect profile of rectally administered misoprostol in the third stage of labor. Obstet Gynecol. 2003 May;101(5 Pt 1):968-74.

Recibido: 04 /08/2015

Aceptado: 19 /11/2015 